\title{
Molecular Structure, Vibrational Analysis and First Order Hyperpolarizability of 4-Methyl-3-Nitrobenzoic Acid Using Density Functional Theory
}

\author{
Jyothi Prashanth1', Gaddam Ramesh'1, Jarupula Laxman Naik1, Jai Kishan 0jha², \\ Byru Venkatram Reddy ${ }^{*}$, Gandham Ramana Rao ${ }^{1}$ \\ ${ }^{1}$ Department of Physics, Kakatiya University, Warangal, India \\ ${ }^{2}$ Department of Physics, Govt. Degree \& P.G. College, Adilabad, India \\ Email: ${ }^{*}$ bvreddy67@yahoo.com
}

Received 16 January 2015; accepted 24 March 2015; published 25 March 2015

Copyright @ 2015 by authors and Scientific Research Publishing Inc.

This work is licensed under the Creative Commons Attribution International License (CC BY).

http://creativecommons.org/licenses/by/4.0/

(c) (i) Open Access

\section{Abstract}

The Fourier Transform Infrared (FTIR) and FT-Raman spectra of 4-methyl-3-nitrobenzoic acid have been recorded in the range $4000-400 \mathrm{~cm}^{-1}$ and $3500-50 \mathrm{~cm}^{-1}$, respectively. The optimized geometry of the molecule, its vibrational frequencies along with corresponding intensities have been computed using the Density Functional Theory (DFT) employing B3LYP/6-311++G basis set. The scaled values of harmonic vibrational frequencies obtained in the computations have been compared with their experimental counter parts. The scaling factors have been refined to reproduce the frequencies with an RMS error of $11.68 \mathrm{~cm}^{-1}$ between the experimental and computed frequencies. The theoretically constructed spectra agree satisfactorily with those of experimental spectra. First order hyperpolarizability constants have also been evaluated.

\section{Keywords}

4-Methyl-3-Nitrobenzoic Acid, Vibrational Spectra, DFT, First Order Hyperpolarizability

\section{Introduction}

During the past two and a half decades, the quantum chemistry community has been trying very hard to develop "a priori" method of predicting vibrational frequencies of a given molecule. As a result, $a b$ initio computations

${ }^{*}$ Corresponding author.

How to cite this paper: Prashanth, J., Ramesh, G., Naik, J.L., Ojha, J.K., Reddy, B.V. and Ramana Rao, G. (2015) Molecular Structure, Vibrational Analysis and First Order Hyperpolarizability of 4-Methyl-3-Nitrobenzoic Acid Using Density Functional Theory. Optics and Photonics Journal, 5, 91-107. http://dx.doi.org/10.4236/opj.2015.53008 
refined to include density functional theory (DFT) have evolved. However, it was found that the values of computed vibrational frequencies using DFT were higher than the corresponding experimental frequencies. This overestimation is attributed to the use of finite basis set, incomplete implementation of the electronic correlation and the neglect of anharmonicity effects [1]. Hence, it became essential to scale the $a b$ initio force fields using spectroscopic data for getting better agreement between the observed and calculated frequencies. In this connection, Pulay's method, which was the basis of scaled quantum mechanical (SQM) force field, became very popular, as it gave transferable scale factors between similar molecules [2]. Yoshida et al. [3] proposed a scaling method, which was an improvement over the method of determining global scale factors for different levels of theory, by a least-squares fit of the calculated frequencies to the experimental frequencies. Sundius [4] modified his MOLVIB program that was capable of computing scale factor calculations accordingly. Researchers in this field, using DFT calculations incorporated into ab initio calculations, have shown that the computed vibrational frequencies and their intensities in the case of many organic molecules agree reasonably well with experimental frequencies on scaling [5]-[7]. But, it is necessary to extend DFT studies to many other molecular systems to prove the applicability of DFT beyond any reasonable doubt. We thought 4-methyl-3-nitrobenzoic acid (MNBA) was one such system.

Benzoic acid and its derivatives have been the subject matter of several investigations [8]-[17]. The reasons are many folds. These are: Benzoic acid occurs widely in plants and animal tissues. It is used in miticides, contrast media in urology, cholocystographic examinations and in the manufacture of pharmaceuticals. The derivatives of benzoic acid are an essential component of the Vitamin B-complex. Further, the herbicidal activity of a molecule containing carboxylic acid group is mainly due to the presence of the acid moiety or a group that is easily convertible to this moiety. Methylnitrobenzoic acid and its derivatives are also known for their local anaesthetic action [16]. Further, it has been demonstrated that 4-Methyl-3-nitrobenzoic acid (MNBA) is a potent inhibitor cancer cell chemotaxis and may be developed into a novel anti-metastatis drug [17]. Hence, we thought it worthwhile to take up a systematic experimental and theoretical investigation of MNBA by recording its Fourier transform Infrared (FTIR) and Fourier transform Raman (FT-Raman) spectra and make a vibrational analysis of its spectra using current level of Density Functional Theory (DFT). Further, it was thought to evaluate first order hyperpolarizability coefficients of MNBA to ascertain its non-linear optical (NLO) behaviour.

\section{Spectral Measurements}

The sample MNBA was obtained from Aldrich Chemical Company, USA and used as such for the spectral measurements. The room temperature Fourier transform infrared (FTIR) spectrum of the compound was recorded using BRUKER IFS-66V FTIR Spectrometer, in the range $4000-400 \mathrm{~cm}^{-1}$ with a scanning speed of 30 $\mathrm{cm}^{-1} \cdot \mathrm{min}^{-1}$ and spectral width $2.0 \mathrm{~cm}^{-1}$. The FT-Raman spectrum of the sample was measured, in the range $3500-50 \mathrm{~cm}^{-1}$ in the Stokes region, using the above Spectrometer equipped with FRA-106 FT-Raman accessory. $1064 \mathrm{~nm}$ line of Nd: YAG laser source operating at $200 \mathrm{mw}$ power was used for excitation. Spectral resolution is believed to be $2 \mathrm{~cm}^{-1}$.

\section{Computational Details}

\subsection{Geometry and Vibrational Frequencies}

Optimization of molecular geometry, evaluation of energy and calculation of vibrational frequencies were carried out, for MNBA, with GAUSSIAN 09W software package [18] implemented on Pentium-V (3.2 GHz) Workstation using Becke's three parameter hybrid functional [19], combined with Lee-Yang-Parr correlation functional [20] (abbreviated as B3LYP). The basis set used was 6-311++G. The SGI grid $(50,194)$ was used for numerical integration. Theoretical force constants in cartesian representation were computed at optimized geometry by assuming $\mathrm{C}_{\mathrm{s}}$ point group symmetry. Scaling of the force constants was made according to scaled quantum mechanical (SQM) procedure [21] [22] employing selective scaling in the natural coordinate representation [23] [24]. Transformation of the force field, normal coordinate analysis, least-square refinement of scale factors, calculation of potential energy distribution (PED) and the evaluation of the IR and Raman intensities were established with the MOLVIB program (version 7.0) written by Suindius [25] [26]. For plotting stimulated IR and Raman spectra, pure Lorentzian band shapes were employed with a band width (FWHM; full width at half maximum) of $10 \mathrm{~cm}^{-1}$. 


\subsection{Raman Intensities}

The Raman activities $\left(S_{i}\right)$ were calculated using GAUSSIAN 09W Program. They were adjusted during the scaling procedure with MOLVIB and subsequently converted to relative Raman intensities $\left(I_{i}\right)$. The following formula, derived from basic theory of Raman scattering [27] [28] was used for this purpose.

$$
I_{i}=f\left(v_{0}-v_{i}\right)^{4} S_{i} /\left\{v_{i}\left[1-\exp \left(h c v_{i} / k T\right)\right]\right\}
$$

where, $v_{0}$ is the exciting frequency (in $\mathrm{cm}^{-1}$ ),

$v_{i}$ is the frequency of the $i^{\text {th }}$ normal mode (in $\mathrm{cm}^{-1}$ ),

$h$ is the Planck's constant,

$c$ is the velocity of light,

$k$ is the Boltzmann's constant and

$f$ is a suitably chosen common normalization factor for all peak intensities.

\subsection{First Order Hyperpolarizability}

The non-linear optical response of an isolated molecule in an electric field can be expressed as a Taylor series expansion of the total dipole moment, $\mu_{\mathrm{t}}$, induced by the field:

$$
\mu_{t}=\mu_{0}+\alpha_{i j} E_{j}+\beta_{i j k} E_{j} E_{k}+\cdots
$$

where, $\mu_{0}$ is the permanent dipole moment,

$\alpha_{i j}$ are the components of polarizability,

$\beta_{i j k}$ are the components of the first order hyperpolarizability.

The first order hyperpolarizability is a third rank tensor. Hence, it contains 27 components represented by a $3 \times$ $3 \times 3$ matrix. Due to Klienman symmetry [29], the 27 components get reduced to 10 components

( $\beta_{x y y}=\beta_{y x y}=\beta_{y y x}=\beta_{y y z}=\beta_{y z y}=\beta_{z y y} ; \ldots$ Similarly other permutation of $x, y, z$ subscripts also take same value). These components are:

$$
\beta_{x x x}, \beta_{x x y}, \beta_{x y y}, \beta_{y y y}, \beta_{x x z}, \beta_{x y z}, \beta_{y y z}, \beta_{x z z}, \beta_{y z z}, \beta_{z z z}
$$

They can be calculated using the following equation [30]:

$$
\beta_{i}=\beta_{i i i}+(1 / 3) \sum_{i \neq j}\left(\beta_{i j j}+\beta_{j i j}+\beta_{i j i}\right)
$$

The total static dipole moment $\mu_{\mathrm{t}}$, the isotropic (or average) linear polarizability $\alpha_{t}$, the anisotropy of polarizability $\Delta \alpha$, and the mean first order hyperpolarizability $\beta_{t}$, using the $x, y, z$ components are defined as:

$$
\begin{gathered}
\mu_{t}=\left(\mu_{x}^{2}+\mu_{y}^{2}+\mu_{z}^{2}\right)^{1 / 2} \\
\alpha_{t}=\left(\alpha_{x x}+\alpha_{y y}+\alpha_{z z}\right) / 3 \\
\Delta \alpha=2^{-1 / 2}\left[\left(\alpha_{x x}-\alpha_{y y}\right)^{2}+\left(\alpha_{y y}-\alpha_{z z}\right)^{2}+\left(\alpha_{z z}-\alpha_{x x}\right)^{2}+6 \alpha_{x x}^{2}\right]^{1 / 2} \\
\beta_{t}=\left(\beta_{x}^{2}+\beta_{y}^{2}+\beta_{z}^{2}\right)^{1 / 2} \\
\text { where, } \beta_{x}=\beta_{x x x}+\beta_{x y y}+\beta_{x z z} \\
\beta_{y}=\beta_{y y y}+\beta_{x x y}+\beta_{y z z} \\
\beta_{z}=\beta_{z z z}+\beta_{x x z}+\beta_{y y z} .
\end{gathered}
$$

\section{Results and Discussion}

\subsection{Ground State Geometry}

The molecule under investigation has two possible rotational conformers resulting from the rotation of the acid group around $\mathrm{C}-\mathrm{C}_{\alpha}$ bond. In the first conformer hydrogen atom of the acid group is nearer to the nitro group in 
the molecular plane. We call this the cis conformer and is shown in Figure 1(a).

In the other conformer, which is a result of rotation of the acid group through $180^{\circ}$ about $\mathrm{C}-\mathrm{C}_{\alpha}$ bond, the hydrogen atom of the acid group is away from the nitrogen group. We call this the trans conformer and is shown in Figure 1(b).

The global minimum energy obtained by DFT structure optimization for cis and trans conformers of MNBA are -664.48076 Hartree and -664.47774 Hartree, respectively. Thus, the energy of the cis conformer is less than the trans conformer in the ground state. Hence, the cis conformer of the MNBA is more stable than its trans conformer. Therefore, subsequent calculations were made using cis conformer only. Numbering, of atoms in the cis conformer of MNBA, is shown in Figure 2.

The optimized geometrical parameters obtained by DFT method employing B3LYP/6-311++G basis set are presented in Table 1.

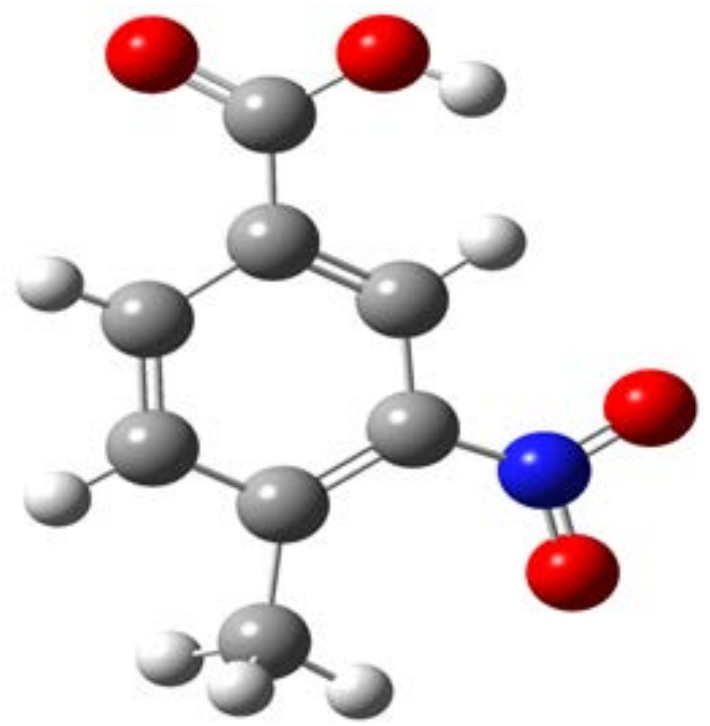

(a)

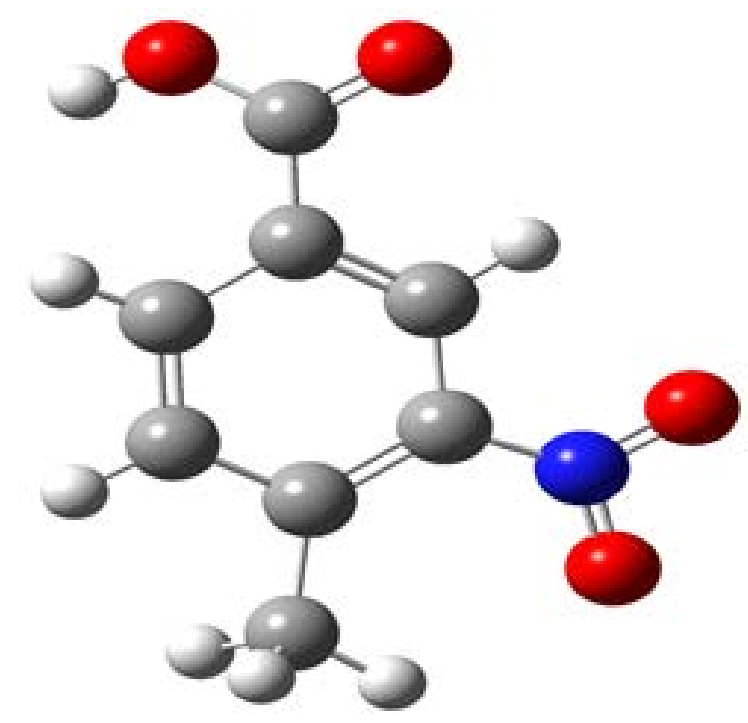

(b)

Figure 1. Conformers of 4-methyl-3-nitrobenzoic acid: (a) cis conformer ( $E=-664.48076$ Hartree); (b) trans conformer ( $E$ $=-664.47774$ Hartree).

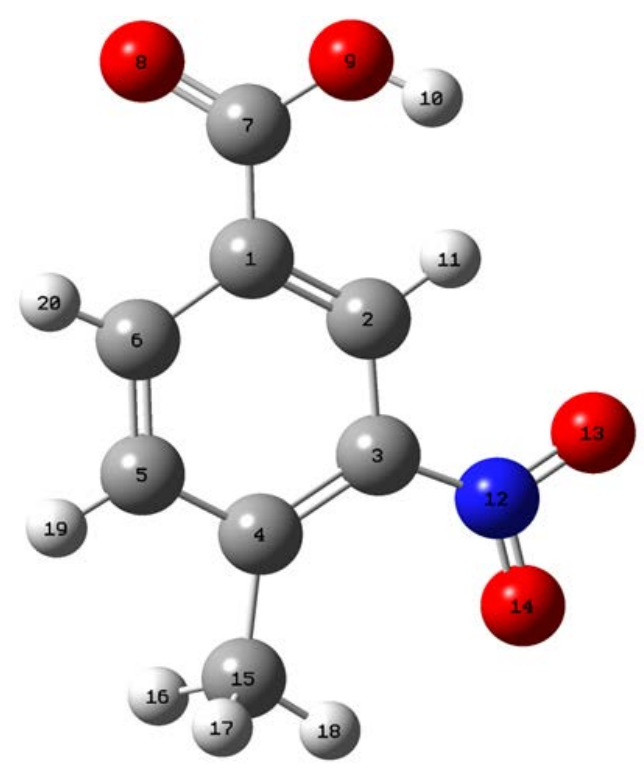

Figure 2. Molecular structure of 4-methyl-3-nitrobenzoic acid with numbering of atoms. 
Table 1. Optimized geometrical parameters of 4-methyl-3-nitrobenzoic acid.

\begin{tabular}{|c|c|c|c|c|c|}
\hline Bond & $\begin{array}{c}\text { Bond length } \\
(\AA)\end{array}$ & Bond angle & $\begin{array}{c}\text { Value } \\
\text { (in degree) }\end{array}$ & Torsional angle & $\begin{array}{c}\text { Value } \\
\text { (in degree) }\end{array}$ \\
\hline C1-C2 & 1.393 & C1-C2-C3 & 120.11 & C1-C2-C3-C4 & -1.0 \\
\hline C2-C3 & 1.397 & C2-C3-C4 & 122.54 & C2-C3-C4-C5 & 1.0 \\
\hline C3-C4 & 1.410 & C3-C4-C5 & 115.78 & C3-C4-C5-C6 & 1.0 \\
\hline C4-C5 & 1.408 & C4-C5-C6 & 122.46 & C3-C2-C1-C7 & -178.0 \\
\hline C5-C6 & 1.388 & C6-C1-C2 & 118.70 & O8-C7-C1-C2 & 157.0 \\
\hline C6-C1 & 1.4058 & C2-C1-C7 & 122.95 & C2-C1-C7-O9 & -22.0 \\
\hline C1-C7 & 1.490 & C1-C7-O8 & 123.19 & C1-C7-O9-H10 & -10.0 \\
\hline O7-C8 & 1.226 & C1-C7-O9 & 117.79 & C6-C1-C2-H11 & 178.0 \\
\hline C7-O9 & 1.384 & C7-C9-H10 & 114.92 & C4-C5-C6-H20 & 178.0 \\
\hline O9-H10 & 0.974 & C1-C2-H11 & 122.46 & C2-C3-N12-O13 & 20.0 \\
\hline C2-H11 & 1.079 & C2-C3-N12 & 115.68 & C2-C3-N12-O14 & -159.0 \\
\hline C3-N12 & 1.466 & C3-N12-O13 & 117.94 & C2-C3-C4-C15 & -179.0 \\
\hline N12-O13 & 1.270 & C3-N12-O14 & 119.00 & C3-C4-C15-H16 & -70.0 \\
\hline N12-O14 & 1.266 & C3-C4-C15 & 125.24 & C3-C4-C15-H17 & 48.0 \\
\hline C4-C15 & 1.507 & C4-C15-H16 & 111.27 & C3-C4-C15-H18 & 169.0 \\
\hline C15-H16 & 1.0904 & C4-C15-H17 & 112.22 & С3-C4-C5-H19 & -179.0 \\
\hline C15-H17 & 1.0897 & C4-C15-C18 & 109.74 & C4-C5-C6-H2O & 179.0 \\
\hline C15-H18 & 1.0894 & C4-C5-H19 & 118.18 & C4-C3-C2-C1 & -1.0 \\
\hline C5-H19 & 1.0812 & C5-C6-H20 & 120.86 & C5-C4-C3-C2 & 1.0 \\
\hline \multirow[t]{3}{*}{ C6-H20 } & 1.0807 & O13-N12-O14 & 123.04 & C6-C5-C4-C2 & 1.0 \\
\hline & & H16-C15-H17 & 106.27 & & \\
\hline & & H17-C15-H18 & 108.70 & & \\
\hline
\end{tabular}

A brief comment may be in order on the effect of the substituent groups, namely the methyl group, the nitro group and the acid group, on the benzene ring of the title molecule. It is important to note that the methyl group is generally considered as electron donating substituent [31] [32], whereas the nitro group is considered as electron withdrawing substituent [33]. Similarly, the acid group is also an electron withdrawing substituent. The underlying chemical mechanisms are hyper conjugation, inductive effect and resonance effect (or mesomeric effect). The methyl group interacts with nearby $\pi$ system via hyper conjugation [33]. In the case of the nitro group [34] and the acid group both the inductive and resonance effects are believed to result in withdrawal of electronic charge from the ring. The three chemical mechanisms mentioned above cause electron delocalization. This is reflected in the values of $\mathrm{C}-\mathrm{C}$ bond distances and $\mathrm{C}-\mathrm{C}-\mathrm{C}$ bond angles of the aromatic ring. Although, $\mathrm{C}-\mathrm{C}$ bond distances and C-C-C bond angles are both affected by substitution, the latter undergoes more pronounced variations (up to several degrees) and, being less affected by systematic errors, are more transferable from one experiment to another. The values of $\mathrm{C}-\mathrm{C}$ bond distances and $\mathrm{C}-\mathrm{C}-\mathrm{C}$ angles of benzene ring presented in Table 1 substantiate this fact, when compared with corresponding values of nitrobenzene and 3-nitrobenzoic acid [35]. It is of particular interest to consider the ipso C2-C3-C4 bond angle, where nitrogen group is substituted at C3 carbon atom of the ring. This ipso bond angle is found to be greater than $120^{\circ}$ (corresponding to $\mathrm{sp}^{2}$ hybridization) for electron withdrawing substituents and smaller than $120^{\circ}$ for electron donating substituents [36]. This is found to be true in the case of MNBA, as DFT level of theory computed the ipso C2-C3-C4 bond angle at $122.54^{\circ}$, since the substituent at C3 atom is the nitro group which is identified earlier as an electron withdrawing group. Further, this value of ipso angle is in excellent agreement with the value of corresponding angle in nitro- 
benzene determined from a regression analysis of the ring angles in the six derivatives of nitrobenzene studied by X-ray crystallography, which yielded a value of $122.7^{\circ}$.

\subsection{Vibrational Assignments}

4-methyl-3-nitrobenzoic acid belongs to $\mathrm{C}_{\mathrm{s}}$ point group symmetry. The molecule consists of 20 atoms. Hence, it has 54 fundamentals, distributed as 36 in-plane vibrations of a' species and 18 out-of-plane vibrations of a" species.

Detailed description of vibrational modes can be made by means of normal coordinate analysis. For this purpose, the full set of 71 primitive (or standard) internal valence coordinates, containing 17 redundancies, was defined as given in Table 2.

By a suitable linear combination of these internal coordinates, a non-redundant set of 54 natural internal coordinates (or local symmetry coordinates) was constructed following the recommendations of Fogarasi et al. [23] [24]. These are summarized in Table 3.

The theoretically calculated DFT force field was transformed to this later set of local symmetry coordinates and used in all subsequently calculations. Observed frequencies (both IR and Raman), calculated frequencies (both un-scaled and scaled), IR and Raman intensities (calculated), potential energy distribution (PED) and vibrational assignment of MNBA are reported in Table 4. The results presented in Table 4 are self-explanatory and the discussion is confined to some of the important modes only.

For visual comparison, the observed and simulated FTIR and FT-Raman spectra of MNBA are presented in Figure 3 and Figure 4, respectively.

The root mean square (rms) deviation between the calculated and experimental frequencies was calculated using following expression in order to determine the goodness of fit.

$$
\mathrm{rms}=\sqrt{\left\{(1 / n-1) \sum_{i=1}^{n}\left[\left(v_{i}\right)^{\text {cal }}-\left(v_{i}\right)^{\text {exp }}\right]^{2}\right\}}
$$

where, $\left(v_{i}\right)^{\text {cal }}$ is the $i^{\text {th }}$ calculated frequency,

$\left(v_{i}\right)^{\exp }$ is the $i^{\text {th }}$ experimental frequency,

and " $n$ " is the number of experimental frequencies.

The rms error between unscaled frequencies and observed frequencies of MNBA was found to be $168.5 \mathrm{~cm}^{-1}$. On using the refined scaling factors, this deviation was reduced to $11.68 \mathrm{~cm}^{-1}$.

\subsubsection{C-C Stretching Vibrations}

The modes 1, 8a, 8b, 14, 19a and 19b are known as C-C stretching vibrations in benzene and its derivatives in Wilson's notation [37]. But mode 1 is highly sensitive to the nature of the substituent and hence classified as ring vibration. Further, in MNBA, mode 14 is observed to have considerable mixing with $\mathrm{C}-\mathrm{H}$ in-plane bending vibration 3 . Hence, only the modes $8 \mathrm{a}, 8 \mathrm{~b}, 19 \mathrm{a}$ and $19 \mathrm{~b}$ are discussed in this section by deferring the discussion of modes 1 and 14 to appropriate sections to a later stage. In the molecule under investigation, modes $8 \mathrm{a}$ and $8 \mathrm{~b}$ are expected around $1600 \mathrm{~cm}^{-1}$. The higher frequency has about 65\% C-C stretching character in MNBA. The remaining PED comes from $\mathrm{C}-\mathrm{H}$ in-plane bending vibration $18 \mathrm{~b}$ and ring vibration $6 \mathrm{~b}$. The lower frequency is a C-C stretching mode to the extent of $51 \%$. It mixes with $C-C^{\prime}$ stretching mode $7 \mathrm{~b}\left(\mathrm{C}^{\prime}\right.$ is atom number 15 in Figure 2), ring vibration 6a and $\mathrm{C}-\mathrm{N}$ stretching vibration 7a. Further, they are known to appear with considerable intensity in the ir spectrum. Hence, the strong absorptions near 1567 and $1621 \mathrm{~cm}^{-1}$ are assigned to C-C stretching vibrations 8a and 8b, respectively. Modes 19a and 19b are expected in the spectral range $1400-1500 \mathrm{~cm}^{-1}$. The higher frequency is a C-C stretching mode to the extent of $56 \%$. This vibration has a good amount of mixing from C-H in-plane bending mode18b in MNBA. The lower frequency exhibits $\mathrm{C}-\mathrm{C}$ stretching character to the extent of $73 \%$ and mixes with C-N stretching vibration $7 \mathrm{a}$. Hence, the band around $1474 \mathrm{~cm}^{-1}$ (scaled calculated value) and ir absorption near $1497 \mathrm{~cm}^{-1}$ are ascribed to modes 19a and 19b, respectively.

\subsubsection{Mode 14 and $\mathrm{C}-\mathrm{H}$ in-Plane Bending Vibrations}

The assignment of mode 14 (the Kekule mode) in which alternate C-C bonds either increase or decrease, is usually difficult as the highest C-H in-plane bending vibration 3 appears in its vicinity around $1300 \mathrm{~cm}^{-1}$. According to the DFT calculations, the bands observed at 1267 and $1296 \mathrm{~cm}^{-1}$ exhibit strong mixing between the 
Table 2. Definition of internal coordinates of 4-methyl-3-nitrobenzoic acid.

\begin{tabular}{|c|c|c|c|}
\hline S. No. & Symbol & Description & Definition \\
\hline \multicolumn{4}{|c|}{ 1) In-plane vibrations (a' species) Stretching } \\
\hline $1-6$ & $d_{i}$ & C-C (ring) & C1-C2, C2-C3, C3-C4, C4-C5, C5-C6, C6-C1 \\
\hline 7 & $r_{i}$ & $\mathrm{C}-\mathrm{C}_{\alpha}$ (acid) & C1-C7 \\
\hline 8 & , & C-C' (methyl) & C4-C15 \\
\hline $9-11$ & , & C-H (ring) & C2-H11, C5-H19, C6-H20 \\
\hline 12 & , & $\mathrm{C}-\mathrm{N}$ & C3-N12 \\
\hline $13-15$ & $R_{i}$ & C-H (methyl) & C15-H16, C15-H17, C15-H18 \\
\hline 16 & $D_{i}$ & $\mathrm{C}_{\alpha}=\mathrm{O}$ & C7-O8 \\
\hline 17 & , & $\mathrm{C}_{\alpha}-\mathrm{OH}$ & C7-O9 \\
\hline 18 & , & $\mathrm{O}-\mathrm{H}$ & O9-H10 \\
\hline $19-20$ & $E_{i}$ & $\mathrm{~N}=\mathrm{O}$ & N12-O13, N12-O14 \\
\hline \multicolumn{4}{|r|}{ Bending } \\
\hline $21-26$ & $\theta_{i}$ & C-C-C (ring) & C6-C1-C2, C1-C2-C3, C2-C3-C4, C3-C4-C5, C4-C5-C6, C5-C6-C1 \\
\hline $27-32$ & $\phi_{i}$ & C-C-H (ring) & C1-C2-H11, C3-C2-H11, C4-C5-H19, C6-C5-H19, C5-C6-H20, C1-C6-H20, \\
\hline $33-35$ & $\varepsilon_{i}$ & C-C-H (methyl) & C4-C15-H16, C4-C15-H17, C4-C15-H18 \\
\hline $36-38$ & $\alpha_{i}$ & $\mathrm{H}-\mathrm{C}-\mathrm{H}$ & H16-C15-H17, H16-C15-H18, H16-C15-H18 \\
\hline 39 & $\lambda_{i}$ & $\mathrm{C}-\mathrm{C}_{\alpha}=\mathrm{O}$ & C1-C7-O8 \\
\hline 40 & , & $\mathrm{C}-\mathrm{C}_{\alpha}-\mathrm{O}$ & C1-C7-O9 \\
\hline 41 & , & $\mathrm{O}-\mathrm{C}_{\alpha}=\mathrm{O}$ & O8-C7-O9 \\
\hline 42 & , & $\mathrm{C}_{\alpha}-\mathrm{O}-\mathrm{H}$ (acid) & C7-O9-H10 \\
\hline $43-44$ & $\beta_{i}$ & C-C-C ${ }_{\alpha}$ (acid) & C6-C1-C7, C2-C1-C7 \\
\hline $45-46$ & , & C-C-C' (methyl) & C3-C4-C15, C5-C4-C15 \\
\hline $47-48$ & , & $\mathrm{C}-\mathrm{C}-\mathrm{N}$ & C2-C3-N12, C4-C3-N12 \\
\hline $49-50$ & $\psi$ & $\mathrm{C}-\mathrm{N}-\mathrm{O}$ & C3-N12-O13, C3-N12-O14 \\
\hline 51 & $\psi_{i}$ & $\mathrm{O}-\mathrm{N}-\mathrm{O}$ & O13-N12-O14 \\
\hline \multicolumn{4}{|c|}{ 1) Out-of-plane vibrations (a" species) Wagging } \\
\hline $52-54$ & $\gamma_{\mathrm{i}}$ & $\pi(\mathrm{CH})$ & C1-C2-C3-C11, C4-C5-C6-C19, C5-C6-C1-H20 \\
\hline 55 & ” & $\pi\left(\mathrm{CC}_{\alpha}\right)$ & C6-C1-C2-C7 \\
\hline 56 & , & $\pi\left(\mathrm{CC}^{\prime}\right)$ & C3-C4-C5-C15 \\
\hline 57 & , & $\pi(\mathrm{CN})$ & C2-C3-C4-N12 \\
\hline 58 & $\omega_{1}$ & $\omega(\mathrm{OH})$ & C1-C7-O9-H10 \\
\hline 59 & ” & $\omega\left(\mathrm{C}_{\alpha}=\mathrm{O}\right)$ & C6-C1-C7-O9 \\
\hline \multicolumn{4}{|r|}{ Torsion } \\
\hline $60-65$ & $\delta_{1}$ & $\tau$ (ring) & $\begin{array}{l}\text { C1-C2-C3-C4, C2-C3-C4-C5, C3-C4-C5-C6, C4-C5-C6-C1, } \\
\text { C5-C6-C1-C2, C6-C1-C2-C3 }\end{array}$ \\
\hline 66 & $\tau_{\mathrm{i}}$ & $\tau\left(\mathrm{CC}_{\alpha}\right)$ & C2-C1-C7-O8 \\
\hline 67 & , & $\tau\left(\mathrm{CC}^{\prime}\right)$ & C3-C4-C15-H18 \\
\hline $68-69$ & ” & $\tau(\mathrm{CN})$ & C2-C3-N12-O14, C4-C3-N12-O13 \\
\hline $70-71$ & $\omega_{1}$ & $\omega\left(\mathrm{NO}_{2}\right)$ & C3-N12-O14-O13, C3-N12-013-O14 \\
\hline
\end{tabular}


Table 3. Definition of local symmetry coordinates of 4-methyl-3-nitrobenzoic acid.

\begin{tabular}{|c|c|c|}
\hline S. No. & Type $^{\mathbf{a}}$ & Definition $^{b}$ \\
\hline $1-6$ & $v(\mathrm{C}-\mathrm{C})$ (ring) & $d_{1}, d_{2}, d_{3}, d_{4}, d_{5}, d_{6}$ \\
\hline 7 & $v\left(\mathrm{C}-\mathrm{C}_{\alpha}\right)$ (acid) & $r_{1}$ \\
\hline 8 & $v(\mathrm{C}-\mathrm{N})$ & $r_{3}$ \\
\hline 9 & $v\left(\mathrm{C}-\mathrm{C}^{\prime}\right)$ (methyl) & $r_{4}$ \\
\hline $10-12$ & $v(\mathrm{C}-\mathrm{H})$ (ring) & $r_{2}, r_{5}, r_{6}$ \\
\hline 13 & $v_{\mathrm{s}}\left(\mathrm{CH}_{3}\right)$ & $\left(R_{1}+R_{2}+R_{3}\right) / \sqrt{3}$ \\
\hline 14 & $v_{\text {as }}\left(\mathrm{CH}_{3}\right)$ & $\left(2 R_{1}-R_{2}-R_{3}\right) / \sqrt{6}$ \\
\hline 15 & $v_{\text {as }}\left(\mathrm{CH}_{3}\right)$ a" & $\left(R_{3}-R_{2}\right) / \sqrt{2}$ \\
\hline 16 & $v(\mathrm{C}=\mathrm{O})$ & $D_{1}$ \\
\hline 17 & $v(\mathrm{C}-\mathrm{O})$ & $D_{2}$ \\
\hline 18 & $v(\mathrm{O}-\mathrm{H})$ & $D_{3}$ \\
\hline 19 & $v_{s}\left(\mathrm{NO}_{2}\right)$ & $\left(E_{1}+E_{2}\right) / \sqrt{2}$ \\
\hline 20 & $v_{\mathrm{as}}\left(\mathrm{NO}_{2}\right)$ & $\left(E_{1}-E_{2}\right) / \sqrt{2}$ \\
\hline $21-23$ & $\beta(\mathrm{CCC})$ (ring) & $\left(\theta_{1}-\theta_{2}+\theta_{3}-\theta_{4}+\theta_{5}-\theta_{6}\right) / \sqrt{6},\left(2 \theta_{1}-\theta_{2}-\theta_{3}+2 \theta_{4}-\theta_{5}-\theta_{6}\right) / \sqrt{12},\left(-\theta_{2}+\theta_{3}-\theta_{5}+\theta_{6}\right) / 2$ \\
\hline $24-29$ & $\begin{array}{c}\beta(\mathrm{CH}) \\
\beta\left(\mathrm{CC}_{\alpha}\right) \\
\beta\left(\mathrm{CC}^{\prime}\right), \beta(\mathrm{CN})\end{array}$ & $\begin{array}{c}\left(\beta_{1}+\beta_{2}+\beta_{3}+\beta_{4}+\beta_{5}+\beta_{6}\right) / \sqrt{6}, \quad\left(\beta_{2}+\beta_{3}-\beta_{5}-\beta_{6}\right) / 2, \quad\left(2 \beta_{1}+\beta_{2}-\beta_{3}-2 \beta_{4}-\beta_{5}+\beta_{6}\right) / \sqrt{12} \\
\left(\beta_{2}-\beta_{3}+\beta_{5}-\beta_{6}\right) / 2, \\
\left(\beta_{1}-\beta_{2}+\beta_{3}-\beta_{4}+\beta_{5}-\beta_{6}\right) / \sqrt{6}, \quad\left(2 \beta_{1}-\beta_{2}-\beta_{3}+2 \beta_{4}-\beta_{5}-\beta_{6}\right) / \sqrt{12}\end{array}$ \\
\hline 30 & $\delta_{\mathrm{s}}\left(\mathrm{CH}_{3}\right)$ & $\left(\varepsilon_{1}+\varepsilon_{2}+\varepsilon_{3}-\alpha_{1}-\alpha_{2}-\alpha_{3}\right) / \sqrt{6}$ \\
\hline 31 & $\delta_{\text {as }}\left(\mathrm{CH}_{3}\right)$ & $\left(2 \alpha_{3}-\alpha_{2}-\alpha_{1}\right) / \sqrt{6}$ \\
\hline 32 & $\delta_{\text {as }}\left(\mathrm{CH}_{3}\right)$ a" & $\left(\alpha_{2}-\alpha_{1}\right) / \sqrt{2}$ \\
\hline 33 & $x\left(\mathrm{CH}_{3}\right)$ & $\left(2 \varepsilon_{1}-\varepsilon_{2}-\varepsilon_{3}\right) / \sqrt{6}$ \\
\hline 34 & $\chi\left(\mathrm{CH}_{3}\right) \mathrm{a}$ & $\left(\varepsilon_{3}-\varepsilon_{2}\right) / \sqrt{2}$ \\
\hline 35 & $\delta\left(\mathrm{C}_{\alpha}=\mathrm{O}\right)$ & $\left(2 \lambda_{2}-\lambda_{1}-\lambda_{3}\right) / \sqrt{6}$ \\
\hline 36 & $\delta\left(\mathrm{C}_{\alpha}-\mathrm{OH}\right)$ & $\left(\lambda_{1}-\lambda_{3}\right) / \sqrt{2}$ \\
\hline 37 & $\delta(\mathrm{OH})$ & $\lambda$ \\
\hline 38 & $\delta\left(\mathrm{NO}_{2}\right)$ & $\left(2 \psi_{3}-\psi_{1}-\psi_{2}\right) / \sqrt{6}$ \\
\hline 39 & $\chi\left(\mathrm{NO}_{2}\right)$ & $\left(\psi_{1}-\psi_{2}\right) / \sqrt{2}$ \\
\hline $40-42$ & $\pi(\mathrm{CH})$ & $\gamma_{2}, \gamma_{5}, \gamma_{6}$ \\
\hline 43 & $\pi\left(\mathrm{CC}_{\alpha}\right)$ & $\gamma_{1}$ \\
\hline 44 & $\pi(\mathrm{CN})$ & $\gamma_{3}$ \\
\hline 45 & $\pi\left(\mathrm{CC}^{\prime}\right)$ & $\gamma_{4}$ \\
\hline $46-48$ & $\tau(\mathrm{CCCC})$ & $\left(\delta_{1}-\delta_{2}+\delta_{3}-\delta_{4}+\delta_{5}-\delta_{6}\right) / \sqrt{6},\left(-\delta_{1}+2 \delta_{2}-\delta_{3}-\delta_{4}+2 \delta_{5}-\delta_{6}\right) / \sqrt{12},\left(\delta_{1}-\delta_{3}+\delta_{4}-\delta_{6}\right) / 2$ \\
\hline 49 & $\omega(\mathrm{OH})$ & $\omega_{1}$ \\
\hline 50 & $\omega\left(\mathrm{C}_{\alpha}=\mathrm{O}\right)$ & $\omega_{2}$ \\
\hline 51 & $\tau\left(\mathrm{CC}_{\alpha}\right)$ & $\tau_{1}$ \\
\hline 52 & $\tau\left(\mathrm{CC}^{\prime}\right)$ & $\tau_{2}$ \\
\hline 53 & $\omega\left(\mathrm{NO}_{2}\right)$ & $\left(\omega_{3}-\omega_{4}\right) / \sqrt{2}$ \\
\hline 54 & $\tau(\mathrm{CN})$ & $\left(\tau_{3}-\tau_{4}\right) / \sqrt{2}$ \\
\hline
\end{tabular}

${ }^{\mathrm{a}}$ The symbols are used for description of the normal modes in PED in Table 4. ${ }^{\mathrm{b}}$ The internal coordinates used here are defined in Table 2. 
Table 4. Observed and B3LYP/6-311++G level calculated vibrational frequencies (in $\mathrm{cm}^{-1}$ ) and vibrational assignments of 4-methyl-3-nitrobenzoic acid.

\begin{tabular}{|c|c|c|c|c|c|c|c|c|}
\hline \multirow{2}{*}{ S. No. } & \multirow{2}{*}{ Mode $^{a}$} & \multicolumn{2}{|c|}{ Obs. freq. $\left(\mathrm{cm}^{-1}\right)$} & \multicolumn{2}{|c|}{ Cal. freq. $\left(\mathrm{cm}^{-1}\right)$} & \multirow{2}{*}{\multicolumn{2}{|c|}{$\operatorname{IR}^{\mathrm{b}}\left(\mathbf{I}_{\mathbf{i}}\right) \operatorname{Raman}^{\mathrm{c}}\left(\mathbf{A}_{\mathrm{i}}\right)$}} & \multirow{2}{*}{ Vibrational assignment $^{d}$} \\
\hline & & IR & Raman & Unscalec & Scaled & & & \\
\hline \multicolumn{9}{|c|}{ Aromatic nucleus-in-plane vibrations } \\
\hline 1 & $v(C-C) 1$ & 655 & 675 & 701 & 691 & 28.75 & 5.88 & $1(58)+12(18)+7 a(12)$ \\
\hline 2 & $v(C-C) 8 a$ & 1567 & 1571 & 1585 & 1594 & 55 & 28.23 & $8 a(51)+7 b(27)+6 a(12)+7 a(11)$ \\
\hline 3 & $v(C-C) 8 b$ & 1621 & 1625 & 1652 & 1658 & 85 & 70.58 & $8 b(65)+18 b(12)+6 b(11)$ \\
\hline 4 & $v(\mathrm{C}-\mathrm{C}) 14$ & 1267 & 1264 & 1254 & 1254 & 87.5 & 24.7 & $14(49)+3(26)+12(10)$ \\
\hline 5 & $v(C-C) 19 a$ & - & - & 1464 & 1474 & 14.16 & 20.61 & $19 a(73)+7 a(19)$ \\
\hline 6 & $v(C-C) 19 b$ & 1497 & 1494 & 1541 & 1520 & 78.75 & 9.411 & $19 b(56)+18 b(23)$ \\
\hline 7 & $v(\mathrm{C}-\mathrm{H}) 2$ & - & 3087 & 3207 & 3083 & 25.12 & 29.41 & 2(99) \\
\hline 8 & $v(\mathrm{C}-\mathrm{H}) 20 \mathrm{a}$ & 3090 & - & 3204 & 3067 & 56.25 & 13.79 & 20a(99) \\
\hline 9 & $v(\mathrm{C}-\mathrm{H}) 20 \mathrm{~b}$ & 3065 & 3067 & 3183 & 3063 & 53.75 & 11.76 & 20b(99) \\
\hline 10 & $v\left(\mathrm{C}-\mathrm{C}_{\alpha}\right) 13$ & 1201 & 1204 & 1176 & 1180 & 40 & 17.64 & $13(38)+3(34)+v\left(\mathrm{C}_{\alpha}-\mathrm{OH}\right)(16)$ \\
\hline 11 & $v(\mathrm{C}-\mathrm{N}) 7 \mathrm{a}$ & 1138 & 1140 & 1160 & 1140 & 45 & 3.52 & $6 a(26)+19 a(18)+7 a(15)+7 b(10)$ \\
\hline 12 & $v\left(C-C^{\prime}\right) 7 b$ & - & - & 1280 & 1293 & 36.8 & 31 & $14(30)+7 b(19)+18 b(15)$ \\
\hline 13 & $\beta(\mathrm{CH}) 3$ & 1296 & 1292 & 1203 & 1275 & 91.25 & 28.53 & $3(35)+12(22)+14(16)$ \\
\hline 14 & $\beta(\mathrm{CH}) 18 \mathrm{a}$ & 1168 & 1168 & 1106 & 1134 & 37.5 & 23.52 & $18 \mathrm{a}(45)+8 \mathrm{a}(18)+6 \mathrm{a}(12)+v\left(\mathrm{C}_{\alpha}-\mathrm{OH}\right)(10)$ \\
\hline 15 & $\beta(\mathrm{CH}) 18 \mathrm{~b}$ & 1090 & 1074 & 1090 & 1084 & 57.05 & 4.7 & $19 \mathrm{~b}(30)+18 \mathrm{~b}(18)+v\left(\mathrm{C}_{\alpha}-\mathrm{OH}\right)(10)$ \\
\hline 16 & $\beta\left(\mathrm{CC}_{\alpha}\right) 15$ & - & - & 268 & 265 & 17.21 & 30.21 & $12(26)+15(24)+9 b(21)+\delta\left(\mathrm{C}_{\alpha}-\mathrm{OH}\right)(13)$ \\
\hline 17 & $\beta(\mathrm{CN}) 9 \mathrm{~b}$ & - & - & 338 & 336 & 21.61 & 22.21 & $9 \mathrm{~b}(32)+\gamma\left(\mathrm{NO}_{2}\right)(24)+\delta\left(\mathrm{C}_{\alpha}-\mathrm{OH}\right)(13)+15(10)$ \\
\hline 18 & $\beta\left(\mathrm{CC}^{\prime}\right) 9 \mathrm{a}$ & - & 343 & 352 & 360 & 2.21 & 8.235 & $9 \mathrm{a}(25)+6 \mathrm{a}(25)+7 \mathrm{a}(19)+\delta\left(\mathrm{C}_{\alpha}-\mathrm{OH}\right)(12)$ \\
\hline 19 & $\beta(\mathrm{CCC}) 6 \mathrm{a}$ & - & 508 & 482 & 478 & 21 & 7.058 & $6 \mathrm{a}(45)+\delta\left(\mathrm{C}_{\alpha}-\mathrm{OH}\right)(23)+\gamma\left(\mathrm{NO}_{2}\right)(18)$ \\
\hline 20 & $\beta(\mathrm{CCC}) 6 \mathrm{~b}$ & - & 377 & 397 & 390 & 2.13 & 20 & $1(31)+6 \mathrm{~b}(19)+9 \mathrm{a}(14)+\delta\left(\mathrm{C}_{\alpha}=\mathrm{O}\right)(10)$ \\
\hline 21 & $\beta(\mathrm{CCC}) 12$ & 805 & 814 & 789 & 789 & 23.75 & 75.29 & $12(31)+\delta\left(\mathrm{NO}_{2}\right)(15)+1(15)+13(11)$ \\
\hline \multicolumn{9}{|c|}{ Aromatic nucleus-out-of-plane vibrations } \\
\hline 22 & $\pi(\mathrm{CH}) 5$ & 830 & 856 & 877 & 871 & 47.5 & 3.52 & $5(84)$ \\
\hline 23 & $\pi(\mathrm{CH}) 11$ & 744 & 759 & 762 & 766 & 81.25 & 4.7 & 11(84) \\
\hline 24 & $\pi(\mathrm{CH}) 17 \mathrm{~b}$ & 948 & - & 957 & 947 & 72.5 & 0.68 & $17 b(65)+16 b(17)$ \\
\hline 25 & $\pi\left(\mathrm{CC}_{\alpha}\right) 10 \mathrm{~b}$ & - & 196 & 204 & 211 & 0.52 & 12.94 & $\omega\left(\mathrm{NO}_{2}\right)(26)+10 \mathrm{~b}(21)+17 \mathrm{a}(18)+\tau\left(\mathrm{CC}_{\alpha}\right)(15)+16 \mathrm{a}(12)$ \\
\hline 26 & $\pi(\mathrm{CN}) 17 \mathrm{a}$ & - & 172 & 158 & 158 & 44.7 & 1.37 & $10 \mathrm{~b}(22)+16 \mathrm{~b}(15)+\omega\left(\mathrm{C}_{\alpha}=\mathrm{O}\right)(20)+17 \mathrm{a}(14)+10 \mathrm{a}(10)$ \\
\hline 27 & $\pi\left(\mathrm{CC}^{\prime}\right) 10 \mathrm{a}$ & - & 291 & 277 & 280 & 22.35 & 1.37 & $10 \mathrm{a}(28)+\omega\left(\mathrm{C}_{\alpha}=\mathrm{O}\right)(20)+16 \mathrm{a}(15)+10 \mathrm{~b}(14)$ \\
\hline 28 & $\tau(\mathrm{CCCC}) 4$ & 699 & 695 & 738 & 728 & 55 & 9.411 & $4(32)+\omega\left(\mathrm{C}_{\alpha}=\mathrm{O}\right)(20)+10 \mathrm{~b}(16)$ \\
\hline 29 & $\tau(\mathrm{CCCC}) 16 \mathrm{a}$ & 560 & - & 574 & 575 & 27.5 & 7.58 & $16 \mathrm{a}(34)+\omega(\mathrm{OH})(12)+10 \mathrm{~b}(12)+17 \mathrm{a}(11)$ \\
\hline 30 & $\tau(\mathrm{CCCC}) 16 \mathrm{~b}$ & 485 & 489 & 445 & 450 & 32.5 & 4.7 & $16 b(52)+17 a(16)+10 b(12)$ \\
\hline \multicolumn{9}{|c|}{ Vibrations of acid group } \\
\hline 31 & $v\left(\mathrm{C}_{\alpha}=\mathrm{O}\right)$ & 1698 & - & 1713 & 1714 & 100 & 1.72 & $v\left(\mathrm{C}_{\alpha}=\mathrm{O}\right)(80)$ \\
\hline 32 & $v\left(\mathrm{C}_{\alpha}-\mathrm{OH}\right)$ & 1319 & 1316 & 1316 & 1313 & 90 & 23.52 & $v\left(\mathrm{C}_{\alpha}-\mathrm{OH}\right)(29)+12(25)+14(22)$ \\
\hline 33 & $\delta(\mathrm{OH})$ & 1425 & - & 1455 & 1459 & 95 & 5.83 & $\delta(\mathrm{OH})(61)+19 \mathrm{~b}(16)$ \\
\hline 34 & $v(\mathrm{O}-\mathrm{H})$ & - & - & 3627 & 3627 & 8.33 & 3.44 & $v(\mathrm{O}-\mathrm{H})(100)$ \\
\hline
\end{tabular}




\begin{tabular}{|c|c|c|c|c|c|c|c|c|}
\hline \multicolumn{9}{|c|}{ Continued } \\
\hline 35 & $\delta\left(\mathrm{C}_{\alpha}=\mathrm{O}\right)$ & 630 & 629 & 636 & 636 & 47.5 & 7.05 & $6 \mathrm{a}(30)+\delta\left(\mathrm{C}_{\alpha}=\mathrm{O}\right)(17)+9 \mathrm{~b}(16)$ \\
\hline 36 & $\delta\left(\mathrm{C}_{\alpha}-\mathrm{OH}\right)$ & - & - & 463 & 471 & 20 & 4.82 & $6 \mathrm{a}(30)+\delta\left(\mathrm{C}_{\alpha}=\mathrm{O}\right)(17)+\delta\left(\mathrm{C}_{\alpha}-\mathrm{OH}\right)(16)$ \\
\hline 37 & $\omega(\mathrm{OH})$ & 908 & 914 & 907 & 922 & 56.25 & 11.76 & $\omega(\mathrm{OH})(55)+4(16)$ \\
\hline 38 & $\omega\left(\mathrm{C}_{\alpha}=\mathrm{O}\right)$ & - & 139 & 140 & 138 & 2.17 & 38.82 & $\omega\left(\mathrm{C}_{\alpha}=\mathrm{O}\right)(62)+\tau(\mathrm{CN})(23)$ \\
\hline 39 & $\tau\left(\mathrm{CC}_{\alpha}\right)$ & - & - & 48 & 43 & 2.16 & 92 & $\tau\left(\mathrm{CC}_{\alpha}\right)(94)$ \\
\hline \multicolumn{9}{|c|}{ Vibrations of methyl group } \\
\hline 40 & $v_{\mathrm{s}}\left(\mathrm{CH}_{3}\right)$ & 2945 & 2936 & 3037 & 2946 & 57.5 & 44.78 & $v_{\mathrm{s}}\left(\mathrm{CH}_{3}\right)(99)$ \\
\hline 41 & $v_{\text {as }}\left(\mathrm{CH}_{3}\right)$ & - & 2977 & 3101 & 2991 & 3.33 & 14.11 & $v_{a s}\left(\mathrm{CH}_{3}\right)(99)$ \\
\hline 42 & $v_{\mathrm{as}}\left(\mathrm{CH}_{3}\right) \mathrm{a} "$ & 2990 & 2991 & 3114 & 3005 & 62.6 & 14.51 & $v_{\text {as }}\left(\mathrm{CH}_{3}(93) \mathrm{a} "\right.$ \\
\hline 43 & $\delta_{\mathrm{s}}\left(\mathrm{CH}_{3}\right)$ & 1370 & 1378 & 1398 & 1384 & 90 & 17.64 & $\delta_{\mathrm{s}}\left(\mathrm{CH}_{3}\right)(82)$ \\
\hline 44 & $\delta_{\text {as }}\left(\mathrm{CH}_{3}\right)$ & 1448 & 1449 & 1405 & 1460 & 56.25 & 17.64 & $\delta_{\mathrm{as}}\left(\mathrm{CH}_{3}\right)(79)+19 \mathrm{a}(17)$ \\
\hline 45 & $\delta_{\text {as }}\left(\mathrm{CH}_{3}\right) \mathrm{a} "$ & - & - & 1515 & 1480 & 4.01 & 4.13 & $\delta_{\text {as }}\left(\mathrm{CH}_{3}\right) \mathrm{a} "(84)$ \\
\hline 46 & $\gamma\left(\mathrm{CH}_{3}\right)$ & 1010 & 1005 & 1028 & 1017 & 17.5 & 3.52 & $\gamma\left(\mathrm{CH}_{3}\right)(52)+\delta_{\mathrm{as}}\left(\mathrm{CH}_{3}\right)(16)$ \\
\hline 47 & $\gamma\left(\mathrm{CH}_{3}\right) \mathrm{a} "$ & - & 1031 & 1034 & 1073 & 5.83 & 2.94 & $\gamma\left(\mathrm{CH}_{3}\right) \mathrm{a} "(66)+\delta_{\mathrm{as}}\left(\mathrm{CH}_{3}\right) \mathrm{a} "(19)$ \\
\hline 48 & $\tau\left(\mathrm{CC}^{\prime}\right)$ & - & 86 & 100 & 99 & 70.58 & 0.68 & $\tau\left(\mathrm{CC}^{\prime}\right)(82)$ \\
\hline \multicolumn{9}{|c|}{ Vibrations of nitro group } \\
\hline 49 & $v_{\mathrm{s}}\left(\mathrm{NO}_{2}\right)$ & 1354 & 1352 & 1352 & 1356 & 85 & 100 & $v_{\mathrm{s}}\left(\mathrm{NO}_{2}\right)(37)+14(19)+7 \mathrm{a}(19)$ \\
\hline 50 & $v_{\text {as }}\left(\mathrm{NO}_{2}\right)$ & 1533 & 1535 & 1523 & 1531 & 97.5 & 32.94 & $v_{\mathrm{as}}\left(\mathrm{NO}_{2}\right)(69)+19 \mathrm{~b}(16)$ \\
\hline 51 & $\delta(\mathrm{NO} 2)$ & 761 & 778 & 745 & 769 & 45 & 4.7 & $1(35)+\delta\left(\mathrm{NO}_{2}\right)(28)+12(14)$ \\
\hline 52 & $\gamma\left(\mathrm{NO}_{2}\right)$ & 532 & - & 513 & 514 & 45 & 6.2 & $\gamma\left(\mathrm{NO}_{2}\right)(31)+6 b(28)+9 b(14)$ \\
\hline 53 & $\omega\left(\mathrm{NO}_{2}\right)$ & - & - & 677 & 665 & 1.21 & 2.06 & $4(54)+\omega\left(\mathrm{NO}_{2}\right)(13)+10 \mathrm{a}(12)$ \\
\hline 54 & $\tau(\mathrm{CN})$ & - & - & 42 & 40 & 0.89 & 2.184 & $\tau(\mathrm{CN})(62)+\tau\left(\mathrm{CC}^{\prime}\right)(25)$ \\
\hline
\end{tabular}

${ }^{a}$ Mode in Wilson's notation [37]; ${ }^{b}$ Relative infrared intensities are normalized to 100 ; ${ }^{\mathrm{C}}$ Raman intensities are normalized to 100 ; ${ }^{\mathrm{d}}$ Number in the parenthesis is \% of PED and number before the parenthesis is vibrational mode. PED less than $10 \%$ is not shown. $v$, stretching; $v_{\mathrm{s}}$, symmetric stretching; $v_{\text {as }}$, asymmmetric stretching; $\beta$, in-plane bending; $\delta$, deformation; $\gamma$, in-plane rocking; $\pi$, out-of-plane bending; $\tau$, torsion; $\omega$, wagging. C ${ }_{\alpha}$, Carbon atom of acid group; C', Carbon atom of methyl group-Not observed.

modes 14 and 3. Further, both of them mix with ring vibration 12 to a considerable extent. Hence, it is difficult to make an unequivocal choice for their assignment. However, going by the highest PED contribution, the ir absorption at $1267 \mathrm{~cm}^{-1}$, which has $49 \%$ C-C stretching character can be attributed to mode 14 . It seems reasonable as this frequency falls in the range $1235-1290 \mathrm{~cm}^{-1}$ proposed for mode 14 by Varsanyi in 1,2,4-tri light substituted benzenes (see Ref. 8, pp 323). The C-H in-plane bending vibrations are designated as mode 3, 18a and 18b in 1,2,4-tri-substituted benzenes. In aromatic compounds they generally appear in the spectral region $1000-1300 \mathrm{~cm}^{-1}$. Consequently, the ir absorption near $1296 \mathrm{~cm}^{-1}$, with its Raman counterpart around 1292 $\mathrm{cm}^{-1}$, which has $35 \% \mathrm{C}-\mathrm{H}$ in-plane bending character is ascribed to mode 3 . Using the results of normal coordinate analysis, modes 18a and 18b are identified near 1168 and $1090 \mathrm{~cm}^{-1}$ in MNBA. Both of them mix with several other vibrations, which can be seen from Table 4.

\subsubsection{Substituent-Sensitive Modes}

The four ring vibrations $1,6 \mathrm{a}, 6 \mathrm{~b}$ and 12 are sensitive to the position and the nature of the substituent, which makes their correlation with corresponding benzene modes very difficult. Hence, they were identified and assigned from careful consideration of their characteristic eigen vector distribution following Patel et al. [38].

Let us consider the modes $6 \mathrm{a}$ and $6 \mathrm{~b}$ corresponding to the benzene band at $606 \mathrm{~cm}^{-1}$. According to normal coordinate treatment, the Raman shifts at 508 and $377 \mathrm{~cm}^{-1}$ are ascribed to modes 6a and 6b, respectively. As is evident from Table 4, they mix with several other modes. In spite of this mixing, these vibrations retain their essential CCC bending character in the appropriate ratio for these modes. Mode 12 occurs near $1010 \mathrm{~cm}^{-1}$ in 


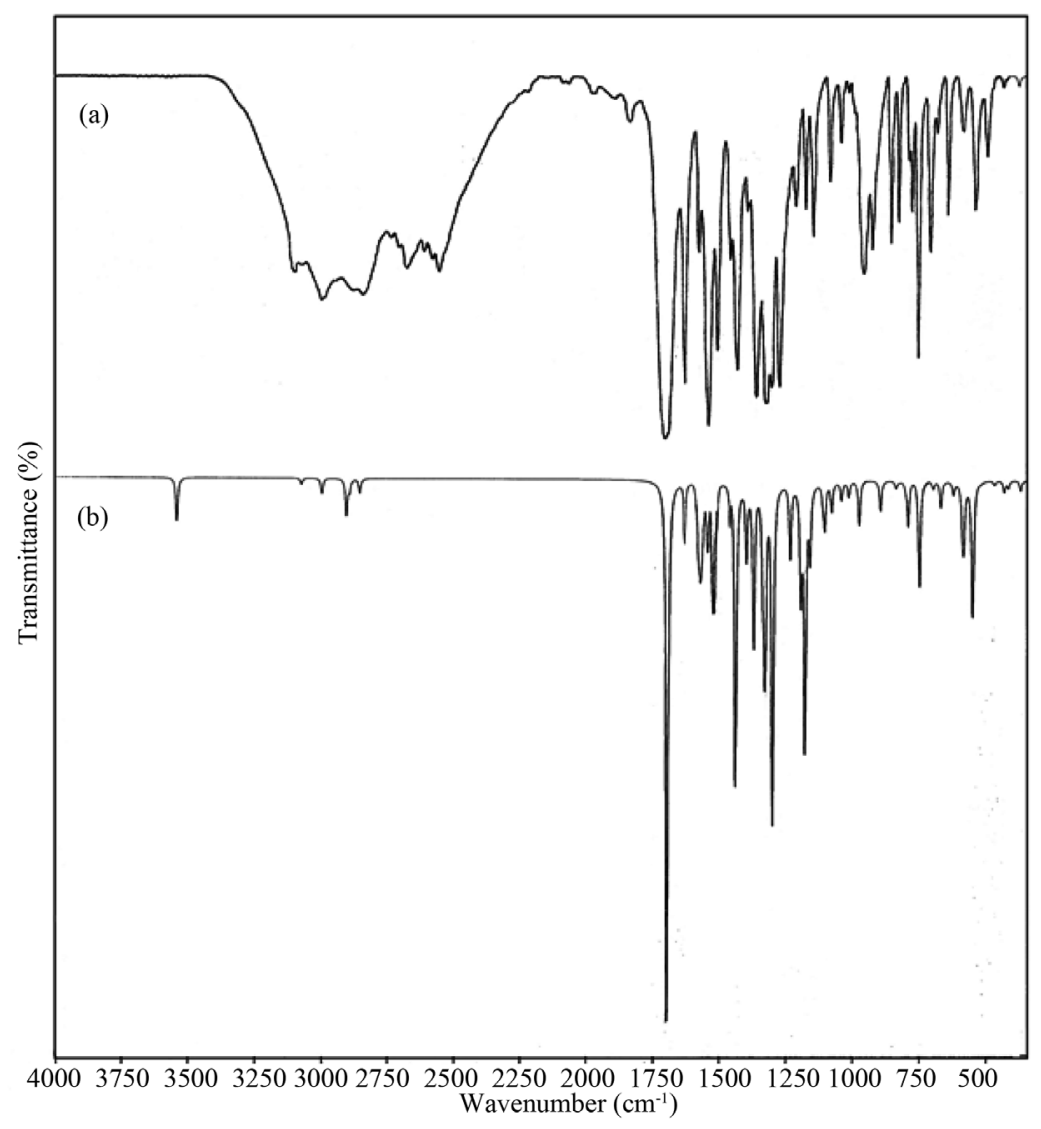

Figure 3. FTIR spectrum of 4-methyl-3-nitrobenzoic acid: (a) Observed; (b) Calculated with B3LYP/6-311++G basis set.

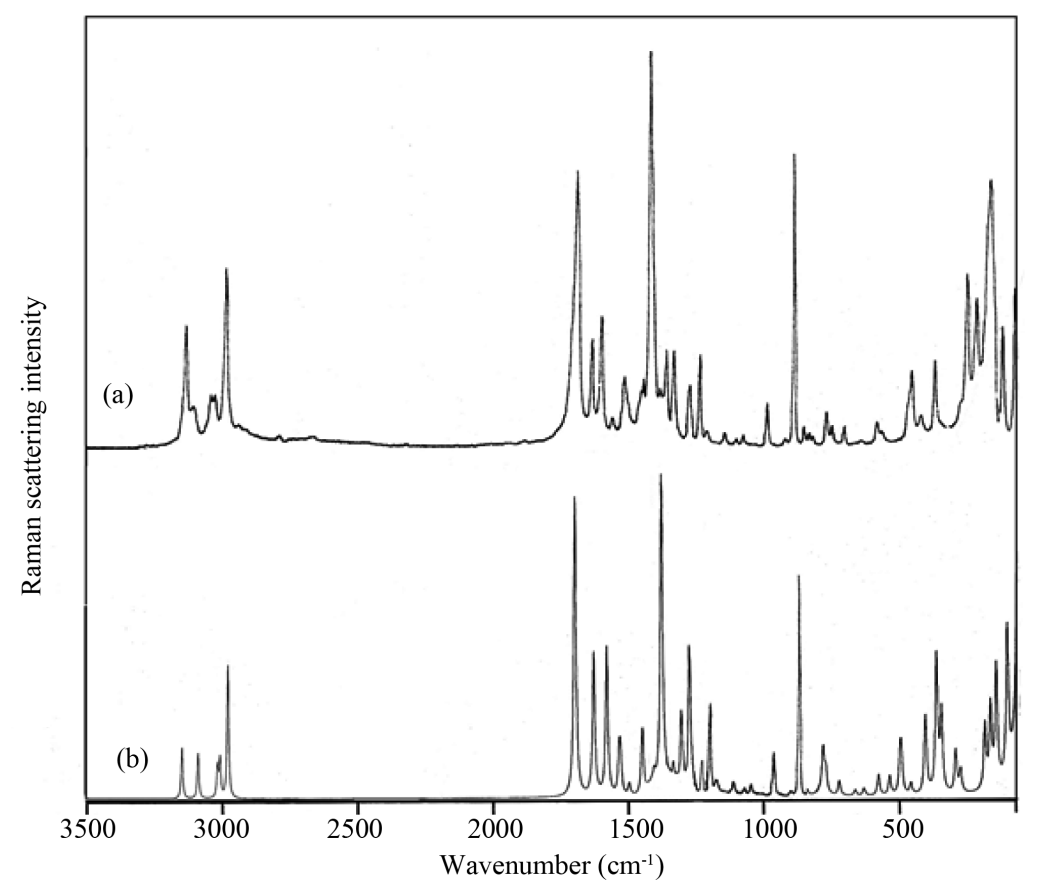

Figure 4. FT-Raman spectrum of 4-methyl-3-nitrobenzoic acid: (a) Observed; (b) Calculated with B3LYP/6-311++G basis set. 
benzene. The only frequency which satisfies the criteria for mode 12 and yet cannot be considered as C-H in-plane bending vibration occurs near $805 \mathrm{~cm}^{-1}$ as strong ir absorption in MNBA. This frequency retains its essential CCC bending nature despite its mixing with several other vibrational frequencies. In benzene, mode 1 at $990 \mathrm{~cm}^{-1}$ is a pure stretching vibration as it is totally symmetric and widely separated from C-H stretching modes. As these restrictions are removed in substituted benzenes, mode 1 can mix with several of the bending modes and also with the lower frequencies of the substituent stretching modes. Hence, a pure mode corresponding to mode 1 of benzene cannot be expected. The only frequency which contains good amount of C-C stretching character and at the same time cannot be considered as $\mathrm{C}-\mathrm{H}$ bending or CCC bending occurs around $655 \mathrm{~cm}^{-1}$ in MNBA. It mixes with several other vibrational modes, as can be seen from Table 4.

\subsection{4. $\mathrm{C}-\mathrm{X}$ [X $=\mathrm{C}_{\alpha}$ (acid), N, C' (Methyl)] Stretching and in-Plane Bending Vibrations}

Modes 13, 7a and 7b are designated as $\mathrm{C}-\mathrm{C}_{\alpha}, \mathrm{C}-\mathrm{N}$ and $\mathrm{C}-\mathrm{C}^{\prime}$ stretching vibrations, whereas the modes $15,9 \mathrm{~b}$ and 9a represent the corresponding in-plane bending vibrations, respectively in MNBA. The ir absorption near 1201 $\mathrm{cm}^{-1}$ has its origin in the stretching vibration of C- $\mathrm{C}_{\alpha}$ bond and assigned to mode 13 in the present molecule under investigation. Mode 13 has $\mathrm{C}_{-} \mathrm{C}_{\alpha}$ stretching character to the extent of $38 \%$ and mixes with mode 3 and $v\left(\mathrm{C}_{\alpha}-\mathrm{OH}\right.$ ). The band near $265 \mathrm{~cm}^{-1}$ (scaled calculated value) is attributed to C- $\mathrm{C}_{\alpha}$ in-plane bending mode 15 . It has $24 \% \mathrm{C}-\mathrm{C}_{\alpha}$ in-plane bending character and mixes with several other fundamentals as reported in Table 4.

The strong ir absorption around $1138 \mathrm{~cm}^{-1}$ is identified as C-N stretching vibration 7a, whereas that near 338 $\mathrm{cm}^{-1}$ (scaled calculated value) is ascribed to the C-N in-plane bending mode $9 \mathrm{~b}$ in MNBA. Their mixed nature can be seen from Table 4.

The band at $1293 \mathrm{~cm}^{-1}$ (scaled calculated value), with $19 \% \mathrm{C}-\mathrm{C}^{\prime}$ stretching nature, is assigned to C-C' stretching mode $7 \mathrm{~b}$. This vibration mixes with C-C stretching vibration 14 and $\mathrm{C}-\mathrm{H}$ in-plane bending mode $18 \mathrm{~b}$. The Raman shift near $343 \mathrm{~cm}^{-1}$ is attributed to C-C' in-plane bending mode 9a. This mode derives 25\% PED from C-C' in-plane bending mode and mixes with several other vibrations as reported in Table 4.

\subsubsection{C-H Stretching Vibrations}

Vibrations 2, 20a and 20b are known as C-H stretching vibrations in the titled molecule under investigation. They generally appear in a narrow spectral region $3000-3100 \mathrm{~cm}^{-1}$ in substituted benzenes. Further, the vibrational pair 20a and 20b, is usually strong in the infrared spectrum, whereas mode 2 is strong in Raman scattering (see Ref. 8, p. 21). Hence, ir bands at 3090 and $3065 \mathrm{~cm}^{-1}$ are assigned to the modes 20a and 20b, respectively, whereas the Raman shift around $3087 \mathrm{~cm}^{-1}$ is attributed to mode 2. According to normal coordinate analysis, as expected, these modes are pure, as each of them has $99 \% \mathrm{C}-\mathrm{H}$ stretching character and do not mix even among themselves.

\subsubsection{C-H Out of Plane Bending Vibrations (Aromatic Nucleus)}

In tri-substituted benzenes, there are three out-of-plane $\mathrm{C}-\mathrm{H}$ bending vibrations designated as modes 5,11 and 17b. The phase relations are: $+2,-1,-1$ for mode $5 ;+1,+1,+1$ for mode $11 ; 0,+2,-2$ for mode $17 \mathrm{~b}$. The + ve and -ve sign indicate increase or decrease, respectively, of internal coordinates involved. Thus, identified ir absorptions near 830, 744 and $948 \mathrm{~cm}^{-1}$ are assigned to the modes 5, 11 and 17b, respectively in MNBA. PED presented in Table 4 shows that the C-H out-of-plane bending character of modes 5 and 11 is $84 \%$, whereas that of $17 \mathrm{~b}$ is $65 \%$. Further, vibrations 5 and 11 do not mix with any other mode, while mode $17 \mathrm{~b}$ mixes with ring torsion vibration $16 \mathrm{~b}$ to the extent of $17 \%$.

\subsubsection{CCCC Torsional Vibrations}

These are also known as ring torsions. There are three of them designated as modes 4, 16a and 16b in benzene and its derivatives. In mode 4, alternate CC torsion angles are either increasing or decreasing. In mode 16a, these angles change in the ratio $+2,-1,-1,+2,-1,-1$, whereas in vibration $16 \mathrm{~b}$, they change in the ratio $0,+2$, $-2,0,+2,-2$. Thus identified, according to the results of normal coordinate analysis, mode 4 appears near 699 $\mathrm{cm}^{-1}$ as a strong ir absorption. It mixes with $\omega\left(\mathrm{C}_{\alpha}=\mathrm{O}\right)$ and mode $10 \mathrm{~b}\left(\mathrm{C}_{\alpha}\right.$ is the carbon atom of the acid group and same as carbon atom number 7 in Figure 2). The absorptions near 560 and $485 \mathrm{~cm}^{-1}$ having $34 \%$ and 52\% ring torsion character are attributed to modes $16 \mathrm{a}$ and $16 \mathrm{~b}$, respectively. Other modes mixing with them can be understood from Table 4. 
4.2.8. C-X $\left[\mathrm{X}=\mathrm{C}_{\alpha}(\right.$ Acid), N, C' (Methyl)] Out-of-Plane Bending Vibrations

In MNBA, C- $\mathrm{C}_{\alpha}$, C-N and C-C' out-of-plane bending vibrations, associated with substituent acid, nitro and methyl groups, are designated as modes 10b, 17a and 10a, respectively. The Raman shifts near 196, 172 and 291 $\mathrm{cm}^{-1}$ are assigned to the modes 10b, 17a and 10a, respectively, on the basis of results of normal coordinate analysis. Table 4 reveals their mixed nature.

\subsubsection{Vibrations of the Acid Group}

There are six in-plane vibrations and three out-of-plane vibrations that have their origin in the acid group of MNBA. These are, $v\left(\mathrm{C}_{\alpha}=\mathrm{O}\right), v\left(\mathrm{C}_{\alpha}-\mathrm{OH}\right), \delta(\mathrm{OH}), v(\mathrm{O}-\mathrm{H}), \delta\left(\mathrm{C}_{\alpha}=\mathrm{O}\right), \delta\left(\mathrm{C}_{\alpha}-\mathrm{OH}\right), \omega(\mathrm{OH}), \omega\left(\mathrm{C}_{\alpha}=\mathrm{O}\right)$ and $\tau\left(\mathrm{CC}_{\alpha}\right)$. The most important characteristic feature of this group is that a very strong ir band appears in the range $1690-1800$ $\mathrm{cm}^{-1}$, that has its origin in the carbonyl stretching vibration of the acid group. Thus, the very strong ir absorption at $1698 \mathrm{~cm}^{-1}$ is assigned to $\mathrm{C}_{\alpha}=\mathrm{O}$ stretching vibration in MNBA. It has $80 \%$ carbonyl stretching character. The stretching and in-plane bending vibrations, $v\left(\mathrm{C}_{\alpha}-\mathrm{OH}\right)$ and $\delta(\mathrm{OH})$, of the acid group generally appear in the range $1200-1450 \mathrm{~cm}^{-1}$ depending on whether monomeric, dimeric or other hydrogen bonded species are present. Usually, $\delta(\mathrm{OH})$ appears at higher frequency than that of $v\left(\mathrm{C}_{\alpha}-\mathrm{OH}\right)$. Moreover, these bands overlap with other bands that are due to aromatic nucleus or aliphatic chain vibrations making unambiguous assignment difficult. On the basis of results of normal coordinate analysis the ir absorption at $1425 \mathrm{~cm}^{-1}$ is found to have $61 \%$ of $\delta(\mathrm{OH})$ character. Hence, it is attributed to $\delta(\mathrm{OH})$. But, it is important to note that it mixes with C-C stretching mode $19 \mathrm{~b}$ of the ring to the extent of $16 \%$. The ir band around $1319 \mathrm{~cm}^{-1}$ having $29 \%$ of $\mathrm{C}_{\alpha}-\mathrm{OH}$ stretching character is attributed to $v\left(\mathrm{C}_{\alpha}-\mathrm{OH}\right)$. It strongly mixes with modes 12 and 14 as can be seen from Table 4 . All benzoic acids, being hydrogen bonded in the solid state, are characterised by a strong ir absorption, in the region $2200-3500 \mathrm{~cm}^{-1}$, attributable to $v(\mathrm{O}-\mathrm{H})$ with a few superimposed maxima that have their origin in C-H stretching vibrations. MNBA is no exception. The centre of the band is around $2970 \mathrm{~cm}^{-1}$. We preferred not to include this in our calculations. The value predicted by DFT near $3627 \mathrm{~cm}^{-1}$ for $v(\mathrm{O}-\mathrm{H})$ corresponds to free molecule in the gaseous state. Assignment of remaining vibrations of this group can be understood from Table 4.

\subsubsection{Vibrations of the Methyl Group}

There are six in-plane and three out-of-plane vibrations that can be associated with the methyl group of MNBA. These are $v_{\mathrm{s}}\left(\mathrm{CH}_{3}\right), v_{\text {as }}\left(\mathrm{CH}_{3}\right), v_{\text {as }}\left(\mathrm{CH}_{3}\right) \mathrm{a}$ ", $\delta_{\mathrm{s}}\left(\mathrm{CH}_{3}\right), \delta_{\text {as }}\left(\mathrm{CH}_{3}\right), \delta_{\text {as }}\left(\mathrm{CH}_{3}\right) \mathrm{a} ", \gamma\left(\mathrm{CH}_{3}\right), \gamma\left(\mathrm{CH}_{3}\right) \mathrm{a}$ and $\tau\left(\mathrm{CC}^{\prime}\right)$. On the basis of the results of normal coordinate analysis, they are assigned to the bands at 2945, 2977R (R indicates Raman shift), 2990, 1370, 1445, 1515C (C indicates scaled calculated value), 1010, 1031R and $86 \mathrm{~cm}^{-1}$, respectively. These results agree with those of other methyl substituted benzenes [39] (and also see Ref.8, pp 395). The important observations that are worth mentioning are:

1) The three C-H stretching vibrations of the methyl group are pure modes as they do not mix with even among themselves and derive 93\% to 99\% PED from the corresponding C-H stretching vibration.

2) The symmetric deformation of the methyl group, along with its out-of-plane asymmetric deformation and methyl torsion should also be considered as pure as they do not mix with any other fundamental vibration. It is to be noted that they derive $82 \%$ - 84\% PED from the relevant vibrational fundamental.

3) The in-plane asymmetric deformation of the methyl group gets predominant part of PED (79\%) from $\delta_{\text {as }}\left(\mathrm{CH}_{3}\right)$. However, it mixes with C-C stretching mode of the ring 19 a to the extent of $17 \%$.

4) The in-plane rocking vibration of the methyl group exhibits $\gamma\left(\mathrm{CH}_{3}\right)$ character to the extent of $52 \%$. It mixes with mode 1 and mode 12 to a considerable extent (see Table 4).

5) The out-of-plane rocking mode of the methyl group has $\gamma\left(\mathrm{CH}_{3}\right) \mathrm{a}$ " nature to the extent of $66 \%$ and mixes with $\delta_{\text {as }}\left(\mathrm{CH}_{3}\right) \mathrm{al}$ to the extent of $19 \%$.

\subsubsection{Vibrations of the Nitro Moiety}

There are four in-plane and two out-of-plane vibrations that can reasonably expected to have their origin in the nitro moiety. These are $v_{\mathrm{s}}\left(\mathrm{NO}_{2}\right), v_{\mathrm{as}}\left(\mathrm{NO}_{2}\right), \delta\left(\mathrm{NO}_{2}\right), \gamma\left(\mathrm{NO}_{2}\right), \omega\left(\mathrm{NO}_{2}\right)$ and $\tau(\mathrm{CN})$.

The symmetric stretching vibration of the nitro group occurs around $1354 \mathrm{~cm}^{-1}$. It has about $37 \%$ symmetric stretching character of the nitro group and acquires additional PED contributions from mode 14 and C-N stretching vibration 7a in MNBA.

The absorption at $1533 \mathrm{~cm}^{-1}$ could be assigned to the asymmetric stretching mode of the nitro group. It has about $69 \%$ of $\mathrm{NO}_{2}$ asymmetric stretching nature and mixes with modes $19 \mathrm{~b}$ to the extent of $16 \%$. 
The band near $761 \mathrm{~cm}^{-1}$ is due to the deformation of the $\mathrm{NO}_{2}$ group to the extent of $28 \%$. It mixes with mode 1 and mode 12 to considerable extent.

The band near $532 \mathrm{~cm}^{-1}$ is mainly due to rocking vibration of the nitro group. However, it has to be described as a mixed mode as there are PED contributions from modes $6 \mathrm{~b}$ and $9 \mathrm{~b}$.

The assignment of $\omega\left(\mathrm{NO}_{2}\right)$ and $\tau(\mathrm{CN})$ can be understood from Table 4.

\subsection{First Order Hyperpolarizability}

Calculation of total molecular dipole moment $\mu_{\mathrm{t}}$ and its components, total molecular first order hyperpolarizability $\beta_{\mathrm{t}}$ and its components of MNBA were made at B3LYP/6-33++G level using GAUSSIAN 09W Program package. The results are summarized in Table 5 .

The first order hyperpolarizability is a measure of non-linear optical (NLO) effects. NLO effects arise due to interaction of incident electromagnetic fields with media (NLO materials). The effect is manifested as generation of new fields that differ in phase, frequency, amplitude or other propagation characteristics that differ from those of the incident fields [40]. NLO effects are important in providing the key functions of frequency shifting, optical modulation, optical switching, optical logic, optical memory for the emerging technologies in the area of telecommunications, signal processing and optical inter-connections [41]-[44]. Hence, DFT has been extensively used to investigate the organic NLO materials [45]-[49].

Urea is a prototypical molecule used in the NLO properties of molecular systems. Hence, it was used frequently as a threshold value for the purpose of comparison. The calculated values of $\mu_{\mathrm{t}}$ and $\beta_{\mathrm{t}}$ for the title compound are 1.5802 Debye and $3.66 \times 10^{-30} \mathrm{~cm}^{5} / \mathrm{esu}$, whereas, the corresponding values for Urea are 1.3732 Debye and $0.3728 \times 10^{-30} \mathrm{~cm}^{5} / \mathrm{esu}$, respectively. Thus we find that the total dipole moment of the title compound is approximately 1.1 times greater than that of Urea and the total first order hyperpolarizability of the title molecules is 9.8 times greater than that of Urea. Hence, it can be concluded, on the basis of the magnitude of the first order hyperpolarizability, the title compound may be a potential applicant in the development of NLO materials.

\section{Conclusion}

A complete vibrational analysis of 4-methyl-3-nitrobenzoic acid is performed using the DFT method at B3LYP/

\begin{tabular}{lc}
$\begin{array}{l}\text { Table 5. Values of dipole moment, } \mu \text { (in Debye) and first order hyperpolarizability, } \beta \text { (in } 10^{-30} \mathrm{~cm}^{5} / \text { e.s.u) } \\
\text { of 4-methyl-3-nitrobenzoic acid. }\end{array}$ \\
\hline Type of component & Value with B3LYP/6-311++G \\
\hline$\mu_{x}$ & 0.883 \\
$\mu_{y}$ & 1.2814 \\
$\mu_{z}$ & -0.5843 \\
$\mu_{t}$ & 1.5802 \\
$\beta_{x x x}$ & -139.047 \\
$\beta_{x x y}$ & -378.159 \\
$\beta_{x y y}$ & 219.875 \\
$\beta_{y y y}$ & -60.230 \\
$\beta_{x x z}$ & 11.4849 \\
$\beta_{x y z}$ & -3.733 \\
$\beta_{y y z}$ & 9.092 \\
$\beta_{x z z}$ & 1.599 \\
$\beta_{y z z}$ & 22.057 \\
$\beta_{z z z}$ & -22.35 \\
$\beta_{t}$ & 3.66 \\
\hline
\end{tabular}


6-311++G level of theory. The effect of methyl-, nitro- and acid groups on the structure of the title compound is discussed. All the fundamental frequencies of the molecule are assigned unambiguously based on the PED and eigenvectors obtained from normal coordinate analysis. The assignment of various vibrational modes is confirmed by the quantitative agreement between the calculated and observed band intensities. It is demonstrated, beyond any reasonable doubt, that the title compound exhibits NLO properties.

\section{Acknowledgements}

The financial support from University Grants Commission, New Delhi, India (F. No. 41-960/2012 (SR), dt.26/7/ 2012) is gratefully acknowledged. The authors are thankful to the Sophisticated Analytical Instrumentation Facility (SAIF), IIT Madras, Chennai, India for the spectral measurements.

\section{References}

[1] Loapez-Token, I., Becucci, M., Pietraperzia, G., Castellucci, E. and Otero, J.C. (2001) Vibrational Spectrum of 4-Fluoroaniline. Journal of Molecular Structure, 565-566, 421-425. http://dx.doi.org/10.1016/S0022-2860(00)00903-0

[2] Pulay, P., Fogarasi, G., Pongor, G., Boggs, J.E. and Vargha, A. (1983) Combination of Theoretical Ab Initio and Experimental Information to Obtain Reliable Harmonic Force Constants. Journal of the American Chemical Society, 105, 7037-7047. http://dx.doi.org/10.1021/ja00362a005

[3] Yoshida, H., Ehara, A. and Massura, H. (2000) Density Functional Vibrational Analysis Using Wavenumber-Linear Scale Factors. Chemical Physics Letters, 325, 477-483. http://dx.doi.org/10.1016/S0009-2614(00)00680-1

[4] Sundius, T. (2002) Scaling of Ab Initio Force Fields by MOLVIB. Vibrational Spectroscopy, 29, 89-95. http://dx.doi.org/10.1016/S0924-2031(01)00189-8

[5] Rauhut, G. and Pulay, P. (1995) Transferable Scaling Factors for Density Functional Derived Vibrational Force Fields. Journal of Physical Chemistry, 99, 3093-3100. http://dx.doi.org/10.1021/j100010a019

[6] Xu, L.C., Li, Z-Y., Tan, W., He, T-J., Liu, F-C. and Chen, D-M. (2005) Density Functional Theory Studies on the Raman and IR Spectra of Meso-Tetraphenylporphyrin Diacid. Spectrochimica Acta Part A: Molecular and Biomolecular Spectroscopy, 62, 850-862. http://dx.doi.org/10.1016/j.saa.2005.03.015

[7] Krishna Kumar, V., John Xavier, R. and Chithambarathanu, T. (2005) Density Functional Theory Study of Vibrational Spectra, and Assignment of Fundamental Vibrational Modes of Succinimide and N-Bromosuccinimide. Spectrochimica Acta Part A: Molecular and Biomolecular Spectroscopy, 62, 931-939.

[8] Varasanyi, G. (1974) Assignments for Vibrational Spectra of Seven Hundred Benzene Derivatives. Vol. 1, Adam Hilger, London, 21, 55, 118, 124, 128, 135, 146, 164, 168, 200, 203, 205, 209, 221, 244, 248, 251, 268, 288, 303, 330331, 334-335, 337-338, 351, 356-358, 380, 382-384, 387, 395, 397-398, 401-402, 405, 412-413, 416, 427, 435.

[9] James, C., Ravikumar, C., Sundius, T., Krishnakumar, V., Kesavamurthy, R., Jayakumar, V.S. and Hubert Joe, I. (2008) FT-Raman and FTIR Spectra, Normal Coordinate Analysis and Ab Initio Computations of (2-Methylphenoxy) Acetic Acid Dimer. Vibrational Spectroscopy, 47, 10-20. http://dx.doi.org/10.1016/j.vibspec.2008.01.006

[10] Furic, K. and Durig, J.R. (1986) Proton-Pair Disorder in Dimers of Aromatic Carboxylic Acids: Vibrational Spectra of Benzoic Acid at Low Temperatures. Chemical Physics Letters, 126, 92-97. http://dx.doi.org/10.1016/0009-2614(86)85122-3

[11] Gunasekaran, S., Natarajan, R.K., Syamala, D. and Rathikha, R. (2006) Normal Coordinate Analysis of Urea Meta Nitro Benzoic Acid Crystal. Indian Journal of Pure and Applied Physics, 44, 315-319.

[12] Sundaraganesan, N., Dominic Joshua, B. and Settu, K. (2007) Vibrational Spectra and Assignments of 5-Amino2-chlorobenzoic Acid by $A b$ Initio Hartree-Fock and Density Functional Methods. Spectrochimica Acta Part A, 66, 381-388. http://dx.doi.org/10.1016/j.saa.2006.03.008

[13] Sundaraganesan, N., Dominic Joshua, B., Meganathan, C., Meenakshi, R. and Cornard, J.P. (2008) Vibrational Spectra and Quantum Chemical Calculations of 3,4-Diaminobenzoic Acid. Spectrochimica Acta Part A, 70, 376-383. http://dx.doi.org/10.1016/j.saa.2007.10.051

[14] Ramalingam, M., Sundaraganesan, N., Saleem, H. and Swaminathan, J. (2008) Experimental (FTIR and FT-Raman) and $A b$ Initio and DFT Study of Vibrational Frequencies of 5-Amino-2-nitrobenzoic Acid. Spectrochimica Acta Part A, 71, 23-30. http://dx.doi.org/10.1016/j.saa.2007.11.016

[15] Sundaraganesan, N., Dominic Joshua, B. and Radjakonmar, T. (2009) Molecular Structure and Vibrational Spectra of 2-Chlorobenzoic Acid by Density Functional Theory and Ab Initio Hartree-Fock Calculations. Indian Journal of Pure and Applied Physics, 47, 248-258.

[16] Tyagi, O.D. and Yadav, M. (1990) Text Book of Synthetic Drugs. Fifth Edition, Anmol Publications, New Delhi, 49. 
[17] Guo, H., Li, M.H., Chen, P., Blake, D.J., Kong, X.N., Hao, X.S., Niu, R.F. and Zhang, N. (2012) 4-Methyl-3-nitrobenzoic Acid, a Migration Inhibitor, Prevents Breast Cancer Metastasis in SCID Mice. Cancer Letters, 324, 232. http://dx.doi.org/10.1016/j.canlet.2011.08.005

[18] Frisch, M.J., Trucks, G.W., Schlegel, H.B., Scuseria, G.E., Robb, M.A., Cheeseman, J.R., et al. (2009) Gaussian, Inc., Wallingford.

[19] Becke, A.D. (1993) Density Functional Thermochemistry. III. The Role of Exact Exchange. The Journal of Chemical Physics, 98, 5648-5652. http://dx.doi.org/10.1063/1.464913

[20] Lee, C., Yang, W. and Parr, R.G. (1998) Development of the Colic-Salvetti Correlation-Energy Formula into a Functional of the Electron Density. Physical Review B, 37, 785-789. http://dx.doi.org/10.1103/PhysRevB.37.785

[21] Berces, A. and Ziegler, T. (1993) Dynamics of Molecules and Chemical Reactions. The Journal of Chemical Physics, 98, 4793-4804.

[22] Pulay, P., Fogarasi, G., Pongor, G., Boggs, J.E. and Vargha, A. (1983) Combination of Theoretical Ab Initio and Experimental Information to Obtain Reliable Harmonic Force Constants. Scaled Quantum Mechanical (SQM) Force Fields for Glyoxal, Acrolein, Butadiene, Formaldehyde, and Ethylene. Journal of the American Chemical Society, 105, 7037-7047. http://dx.doi.org/10.1021/ja00362a005

[23] Fogarasi, G., Pulay, P. and Durig, J.R., Eds. (1985) Chapter 3, Vibrational Spectra and Structure. Elsevier, Amsterdam, Vol. 14, 125.

[24] Fogarasi, G., Zhou, X., Tayler, P.W. and Pulay, P. (1992) The Calculation of Ab Initio Molecular Geometries: Efficient Optimization by Natural Internal Coordinates and Empirical Correction by Offset Forces. Journal of the American Chemical Society, 114, 8191-8201. http://dx.doi.org/10.1021/ja00047a032

[25] Sundius, T. (1990) Molvib-A Flexible Program for Force Field Calculations. Journal of Molecular Structure, 218, 321-326. http://dx.doi.org/10.1016/0022-2860(90)80287-T

[26] Sundius, T. (2002) Scaling of Ab Initio Force Fields by MOLVIB. Vibrational Spectroscopy, 29, 89-95. http://dx.doi.org/10.1016/S0924-2031(01)00189-8

MOLVIB (V.7.0), Calculation of Harmonic Force Fields and Vibrational Modes of Molecules, QCPE Program No. 807, 2002.

[27] Keresztury, G., Holly, S., Varga, J., Besenyei, G., Warng, A.Y. and Durig, J.R. (1993) Vibrational Spectra of Monothiocarbamates-II. IR and Raman Spectra, Vibrational Assignment, Conformational Analysis and Ab Initio Calculations of S-Methyl-N,N-dimethylthiocarbamate. Spectrochimica Acta Part A, 49, 2007-2017, 2019-2026. http://dx.doi.org/10.1016/S0584-8539(09)91012-1

[28] Keresztury, G., Chalmers, J.M. and Griffth, P.R., Eds. (2002) Raman Spectroscopy: Theory in Handbook of Vibrational Spectroscopy. Volume 1, John Wiley and Sons Ltd., New York, 71.

[29] Klienman, D.A. (1962) Nonlinear Dielectric Polarization in Optical Media. Physical Review, 126, 1977-1979. http://dx.doi.org/10.1103/PhysRev.126.1977

[30] Zhang, R., Du, B., Sun, G. and Sun, Y.X. (2002) Experimental and Theoretical Studies on o-, $m$ - and $p$-Chlorobenzylideneaminoantipyrines. Spectrochimica Acta Part A, 75, 1115-1124. http://dx.doi.org/10.1016/j.saa.2009.12.067

[31] Abe, T., Saito, H., Niikura, Y. and Nakano, Y. (2001) Embryonic Development Assay with Daphnia magna: Application to Toxicity of Aniline Derivatives. Chemosphere, 45, 487-495. http://dx.doi.org/10.1016/S0045-6535(01)00049-2

[32] Lipkowitz, K.B. (1982) A Reassessment of Nitrobenzene Valence Bond Structures. Journal of the American Chemical Society, 104, 2647-2648. http://dx.doi.org/10.1021/ja00373a057

[33] Altun, A., Golcuk, K. and Kumru, M. (2003) Theoretical and Experimental Studies of Vibrational Spectra of m-Methylaniline. Journal of Molecular Structure, 625, 17-24.

[34] Politzer, P., Abrahmsen, L. and Sjoberg, P. (1984) Theoretical Organic Chemistry. Journal of the American Chemical Society, 106, 855-860. http://dx.doi.org/10.1021/ja00316a005

[35] Domenicano, A., Schultz, G., Harigittew, I., Colapietro, M., Portalone, G., George, P. and Bock, C.W. (1989) Molecular Structure of Nitrobenzene in the Planar and Orthogonal Conformations: A Concerted Study by Electron Diffraction, X-Ray Crystallography, and Molecular Orbital Calculations. Structural Chemistry, 1, 107-122.

[36] Brunvoll, J., Samdal, S., Thomassen, H., Vilkov, L.V. and Volden, H.V. (1990) The Molecular Structure of Iodobenzene and $p$-Iodonitrobenzene in the Gaseous State. Acta Chemica Scandinavica, 44, 23-30. http://dx.doi.org/10.3891/acta.chem.scand.44-0023

[37] Wilson, E.B. (1934) The Normal Modes and Frequencies of Vibration of the Regular Plane Hexagon Model of the Benzene Molecule. Physical Review, 45, 706-714. http://dx.doi.org/10.1103/PhysRev.45.706

[38] Patel, N.D., Kartha, V.B. and Narasimham, N.A. (1973) Vibrational Spectra Dihalogenated Benzenes. I. In-Plane Vibrations. Journal of Molecular Spectroscopy, 48, 185-201. http://dx.doi.org/10.1016/0022-2852(73)90185-9 
[39] La Lau, C. and Snyder, R.G. (1971) A Valence Force Field for Alkyl Benzenes Toluene, p-Xylene, m-Xylene Mesitylene, and Some of Their Deuterated Analogues. Spectrochimica Acta Part A, 27, 2073-2088. http://dx.doi.org/10.1016/0584-8539(71)80105-8

[40] Sun, Y.X., Hao, Q.L., Wei, W.X., Yu, Z.X., Lu, L.D., Wang, X. and Wang, Y.S. (2009) Experimental and Density Functional Studies on 4-(3,4-Dihydroxybenzylideneamino)antipyrine, and 4-(2,3,4-Trihydroxybenzylidene-amino) Antipyrine. Journal of Molecular Structure: THEOCHEM, 904, 74-82.

[41] Andraud, C., Brotin, T., Garcia, C., Pelle, F., Goldner, P., Bigot, B. and Collet, A. (1994) Theoretical and Experimental Investigations of the Nonlinear-Optical Properties of Vanillin, Polyenovanillin, and Bisvanillin Derivatives. Journal of the American Chemical Society, 116, 2094-2102. http://dx.doi.org/10.1021/ja00084a055

[42] Geskin, V.M., Lambert, C. and Bredas, J.L. (2004) Origin of High Second- and Third-Order Nonlinear Optical Response in Ammonio/Borato Diphenylpolyene Zwitterions: The Remarkable Role of Polarized Aromatic Groups. Journal of the American Chemical Society, 125, 15651-15658. http://dx.doi.org/10.1021/ja035862p

[43] Nakano, M., Fujita, H., Takahata, M. and Yamaguchi, K. (2002) Theoretical Study on Second Hyperpolarizabilities of Phenylacetylene Dendrimer: Toward an Understanding of Structure-Property Relation in NLO Responses of Fractal Antenna Dendrimers. Journal of the American Chemical Society, 124, 9648-9655. http://dx.doi.org/10.1021/ja0115969

[44] Sajan, D., Joe, H., Jayakumar, V.S. and Zaleski, J. (2006) Structural and Electronic Contributions to Hyperpolarizability in Methyl p-Hydroxy Benzoate. Journal of Molecular Structure, 785, 43-53.

[45] Sun, Y.X., Hao, Q.L., Yu, Z.X., Wei, W.X., Lu, L.D. and Wang, X. (2009) Experimental and Density Functional Studies on 4-(4-Cyanobenzylideneamino) Antipyrine. Molecular Physics, 107, 223-235. http://dx.doi.org/10.1080/00268970902769471

[46] Ahmed, A.B., Feki, H., Abid, Y., Boughzala, H., Minot, C. and Mlayah, A. (2009) Crystal Structure, Vibrational Spectra and Theoretical Studies of L-Histidinium Dihydrogen Phosphate-Phosphoric Acid. Journal of Molecular Structure, 920, 1-7.

[47] Abraham, J.P., Sajan, D., Shethigar, V., Dharmaprakash, S.M., Nemec, I., Joe, I.H. and Jayakumar, V.S. (2009) Efficient $\pi$-Electron Conjugated Push-Pull Nonlinear Optical Chromophore 1-(4-Methoxyphenyl)-3-(3,4-dimethoxyphenyl)2-propen-1-one: A Vibrational Spectral Study. Journal of Molecular Structure, 917, 27-36.

[48] Sagdinc, S.G. and Esme, A. (2010) Theoretical and Vibrational Studies of 4,5-Diphenyl-2-2 Oxazole Propionic Acid (Oxaprozin). Spectrochimica Acta Part A, 75, 1370-1376. http://dx.doi.org/10.1016/j.saa.2010.01.004

[49] Ahmed, A.B., Feki, H., Abid, Y., Bonghzala, H. and Minot, C. (2010) Crystal Studies, Vibrational Spectra and NonLinear Optical Properties of L-Histidine Chloride Monohydrate. Spectrochimica Acta Part A, 75, 293-298. http://dx.doi.org/10.1016/j.saa.2009.10.026 